\title{
Training and Application of Radial-Basis Process Neural Network Based on Improved Shuffled Flog Leaping Algorithm
}

\author{
ZHANG QIANG ${ }^{1, \text { a }}$, LIU Li-jie \\ ${ }^{1}$ School of Computer and Information Technology,Northeast Petroleum \\ University, Da qing 163318, China \\ ${ }^{2}$ School of Information Technology,HLJ August First Land Reclamation \\ University, Da qing 163319, China \\ adqpi_zq@163.com bynd_llj@163.com
}

\begin{abstract}
.
A radial basis process neural networks can be established ,which is based on expanding the traditional radial basis function neural network to the time domain. Combined with the excellent characteristics of cloud model transformation between qualitative and quantitative , a improved shuffled flog leaping algorithm based on cloud model theory is presented. It is applied to training the radial basis process neural network. The neural network after optimization is used in pumping unit fault diagnosis. The diagnostic results between the CCHSFLA and BP algorithm were compared. The conclusion is that the RBPNN based on CCHSFLA has better training performance, faster convergence rate and higher accuracy.
\end{abstract}

Keywords: Radial-basis process neural network;Shuffled frog leaping algorithm;Learning; algorithm;fault diagnosis

\section{Introduction}

A radial basis process neural networks model was proposed, which is a kind of three-layer forward structure constituted of input layer, radial basis function hidden layer and output layer. The transformation from input layer to hidden layer is nonlinear and that from hidden layer to output layer is linear. The neurons of hidden layer perform the pattern matching of process input information and aggregation operation of time and respond to the input patterns. A improved shuffled flog leaping algorithm is presented based on cloud model theory. The idea is to initialize the population through reverse learning mechanism, to solve better value around the global best individual and subgroup optimal individual in SFLA by the normal cloud particle operator. Finally, the individual were mutation 
to jump out of local optimal solution by using the theory of chaos. It is applied to training Network structure, which has made very good prediction result.

\section{Radial-basis process neural network model}

\subsection{Radial-Basis Process Neuron Network Model}

Suppose that the input layer of the radial-basis process neural network has $n$ nodes for completing the input of the network of time-varying functions; the middle radial-basis process neuron hidden layer has m nodes, the transformation function of each unit is the radial-basis kernel function; the network output is the linear weighted summary of the output signals of hidden layer nodes[1]. The topological structure of the network is shown in Fig.1.

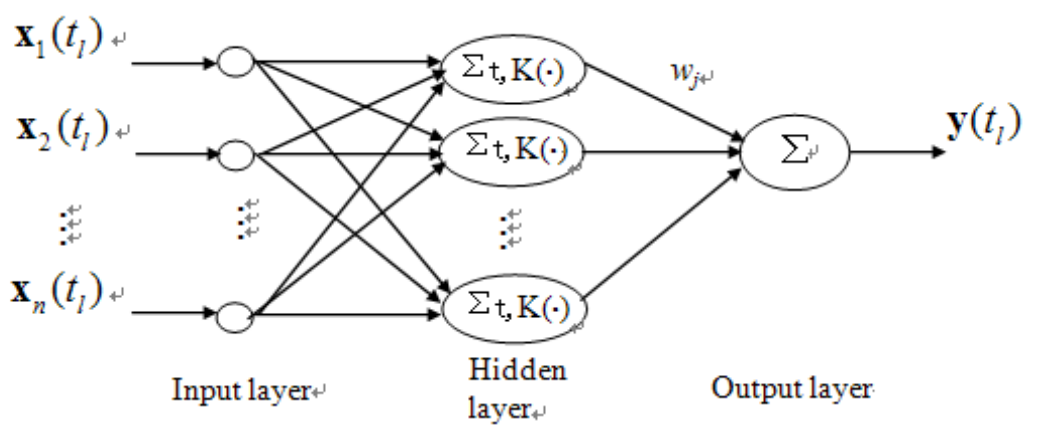

Fig 1 RBF process neural network

In Fig.1, wj $(j=1,2, \ldots, m)$ is the weight coefficient of the output layer, and is an adjustable parameter of the network.Suppose that $\mathrm{X}(\mathrm{t})=(\mathrm{x} 1(\mathrm{t}), \mathrm{x} 2(\mathrm{t}), \ldots, \mathrm{xn}(\mathrm{t}))$ is the input function of the network, $X j(t)$ is the kernel center function of the $j$ th radial-basis process neuron; then the input-output relationship of radial-basis process neural networks is

$$
F\left(\mathbf{X}\left(t_{l}\right)\right)=\sum_{j=1}^{m} w_{j} \sum_{l=1}^{T}\left(\left\|\mathbf{X}\left(t_{l}\right)-\mathbf{X}^{j}\left(t_{l}\right)\right\|\right) \Delta t
$$

\subsection{Learning Algorithm}

Suppose that the input functions and the kernel center functions of RBF process neural networks belong to $(\mathrm{C}[0, \mathrm{~T}]) \mathrm{n}$. The network training mainly includes the adjustment of property parameters in the radial-basis kernel function $\mathrm{K}(\cdot)$, the determination of the radial-basis kernel center function $\mathrm{Xj}(\mathrm{t})$, and the iteration and modification of weight coefficients in the output layer. The network is trained to satisfy the input-output mapping relationship of training samples by way of teacher demonstration. 
For $\boldsymbol{X}(t) \in(C[0, T])^{n}$, define:

$$
\left\|\mathbf{X}\left(t_{l}\right)-\mathbf{X}^{j}\left(t_{l}\right)\right\|=\left(\left(\mathbf{X}\left(t_{l}\right)-\mathbf{X}^{j}\left(t_{l}\right)\right)\left(\mathbf{X}\left(t_{l}\right)-\mathbf{X}^{j}\left(t_{l}\right)\right)^{T}\right)^{\frac{1}{2}}
$$

Give $K$ learning samples $\left(x_{k 1}(t), x_{k 2}(t), \ldots, x_{k n}(t), d_{k}\right)$ for $k=1,2, \ldots, K$, where $d_{k}$ is the expected output of the $k$ th sample in process interval $x_{n}(t)$. Suppose that the actual output of the $k$ th sample is $y_{k}$, the network error function is defined as: $E=\sum_{k=1}^{K}\left(\mathbf{y}_{k}-\mathbf{d}_{k}\right)^{2} \quad$ (3) ;If the radial-basis kernel function is chosen as a Gauss function, that is: $K(v)=\exp \left(-\frac{v^{2}}{2 \sigma^{2}}\right) \quad$ (4) ; where $\sigma$ is called the mean deviation of $m$ kernel center functions which can be determined by the network training of the learning sample set or by the following formulas: $\sigma=d / \sqrt{2 m}$

$$
\text { (5) } ; d=\frac{1}{m}\left(\sum_{j=1}^{m} \sum_{l=1}^{T}\left(\mathbf{X}^{j}\left(t_{l}\right)-\frac{1}{m} \sum_{j=1}^{m} \mathbf{X}^{j}\left(t_{l}\right)\right)^{2} \Delta t\right)^{\frac{1}{2}}
$$

So, the $w_{j}$ is for adjustment parameters. Aimed to the problems that gradient descent method need to calculate the complex gradient information and sensitive to initial values. A Bloch Quantum Evolutionary Algorithm is presented

\section{Cloud Chaos Hypermutation Shuffled Flog Leaping Algorithm(CCHSFLA)}

\subsection{Population initialization}

Shuffled frog leaping algorithm ${ }^{[2]}$ starts iterative calculation from the initial random value, If individuals began calculating from the initial solution which is close to the optimal value, it can accelerate the convergence rate in part. In 2005 Tizhoosh proposed a kind of reverse learning mechanism of machine learning method. The basic idea is to also consider the current estimate and inverse estimate the value of variables, and by comparing the obtained current optimal approximate value. Literature [3] proof: Estimates Based on the reverse are more likely to close the current estimates than random estimate, Specific steps are as follows:

Step 1 An initial solution is generated by random number in $D$ dimension space;

Step 2 Using the formula $X_{i}{ }^{\prime}=a_{i}+b_{i}-x_{i}$ calculate reverse solution of $X$ $X^{\prime}=\left[x_{1}{ }^{\prime}, x_{2}{ }^{\prime}, \ldots, x_{D}{ }^{\prime}\right]$; 
Step 3 Calculate fitness function $f(\bullet)$ of these two solutions, In order to minimum value as an example, if $f\left(X^{\prime}\right)<f(X)$. Use $X^{\prime}$ instead of $X$, Otherwise, continue to use $X$.

\subsection{Optimal values of Sub frogs swarm and the global optimal evolutionary mechanism improvement}

The cloud model which is uncertain transformation model of a kind of qualitative knowledge description and qualitative concept and quantitative values is proposed by Li Deyi .there are the characteristics of uncertainty with certainty, stability and changes in the knowledge expression ${ }^{[4,5]}$. The digital characteristics of the cloud be described by Ex (Expected Value), En (Entropy) and He (Hyper Entropy). Classic shuffled frog leaping algorithm will divide the population into several sub group, Subgroup individuals are updated location reference subgroup optimal individual, If did not improve, it are updated location reference the global optimal individuals . The population is re grouping after internal iterative times. The algorithm optimization speed is fast at the early stage, But these reference values may be not change after iterated several iterations in the late period, cause to decrease the speed, easy to fall into local optimum. According to the principles of sociology, the surrounding of outstanding individuals exist more outstanding individual, Therefore, we improved the way of updating the two optimal value, They are used as matrix respectively, cloud model is used to generate a plurality of new individual. Comparison of these individual if there are better individuals, if there is to replace the original optimal values, and then update the other individual position optimization. Make the body as the cloud model expectations En , Cloud droplets are generated through two times of normal random,the realization of the method is as follows:

Step 1 To generate a normal random number $E n$ ' which the $E n$ is the expected value, $\mathrm{He}$ is standard deviation;

Step 2 To generate a normal random number $X$ which the absolute value of $E x$ is the expected value, $E n^{\prime}$ is standard deviation, $X$ is called a cloud theory in the domain space;

Step 3 Calculate $y=\exp \left(\frac{-(x-E x)^{2}}{2\left(E n^{\prime}\right)^{2}}\right)$, Let $y$ belongs certainty degree of qualitative concept ;

Step 4 Repeat steps $1 \sim$ step 3 until produce $n$ cloud droplets ;

Step 5 Compare the current optimal value fitness value with fitness value corresponding to the $\mathrm{N}$ cloud droplets, Take the best is the current optimal value;

Step 6 Other individuals begin iterative calculation in the original way of classical evolutionary. 


\subsection{Individual variation method based on Chaos Theory}

There are many mutations and leap process excepte smooth and continuous, gradual change for many natural things . It facilitates the generation of new species. In the standard shuffled frog leaping algorithm grouping method, Fitness relatively poor individuals are divided in the last group, the worst individual of the group learn effect than in front of the group, The grouping result has certain limitations of individual learning, But the characteristics of chaos theory ${ }^{[6]} \mathrm{can}$ make up the limitation , to avoid falling into local optimal solution. The following specific variation mode:

Step 1 First select the last sub populations after the packet, According to the formula count $=M \sin \left(\frac{l}{L} \pi\right)$ calculate count,$M$ is the number of frogs in sub populations, $L$ is the total number of iterations, $l$ Is the current number of iterations, Select the count individuals in the last subgroups as the mutation individuals;

Step 2 The individual is metamorphosised by Logistic chaotic sequences $L_{j+1}=\mu L_{j}\left(1-L_{j}\right) ; \mu=4$

Step 3 Use reverse learning mechanism to solve the fitness of reverse solution of these individuals;

Step 4 Comparison Fitness of step 2 and step 3, Take the best as the mutated individual.

\section{Application of the oil wells fault diagnosis}

In the actual data processing, select 147 dynamometer samples(normal, sanding, traveling valve leakage, touch the pump, Knot wax thick, discharge leakage, breakage of a sucker rod and tubing leakage) of different working state of the same type of pumping well. The training set is composed of 105 samples, The test set is composed of 42 samples. The sample data is composed of load and displacement of oil well in one work period, The sampling points for 100, the load and displacement data were fitted time function. Compare the diagnostic performance with BP-PNN, BP-RBPNN and CCHSFLA -RBPNN. Network structure is $2-m-1,2$ input nodes, the input for the displacement time curve and load time curve, The hidden layer neurons has $m$ Radial-basis process neural node, 1 non time varying neuron output node; hidden layer $m=12$, basis fuction is Walsh fuction,when $L=16$,it meet the input requirements of 0.01 of the fitting precision, The error precision $\varepsilon=0.5$; the maximum times of learning $M=10000$; each algorithm was run 10 times, comparing the optimization results as shown in Table 1:

Table 1 Comparison of optimization result

\begin{tabular}{c|c|c|c|c|c}
\hline Algorithm & Minimum & Maximum & Average & Convergence & Average \\
\hline
\end{tabular}




\begin{tabular}{c|c|c|c|c|c}
\hline & error & error & error & & time (s) \\
\hline BP-PNN & 0.3581 & 0.4676 & 0.4165 & 10 & 64.15 \\
\hline BP-RBPNN & 0.2519 & 0.3462 & 0.2857 & 10 & 39.41 \\
\hline $\begin{array}{c}\text { CCHSFLA } \\
\text { - RBPNN }\end{array}$ & 0.1078 & 0.2958 & 0.1756 & 10 & 31.46 \\
\hline
\end{tabular}

Table 2 Comparison of discriminant results

\begin{tabular}{c|c|l}
\hline Network model & $\begin{array}{c}\text { The training sample } \\
\text { set } \\
\text { discrimination rate }\end{array}$ & $\begin{array}{l}\text { The test data } \\
\text { set } \\
\text { classification } \\
\text { rate }\end{array}$ \\
\hline BP-PNN & $87.6 \%$ & $85.7 \%$ \\
\hline BP-RBPNN & $94.9 \%$ & $88.5 \%$ \\
\hline $\begin{array}{c}\text { CCHSFLA - } \\
\text { RBPNN }\end{array}$ & $98.4 \%$ & $93.6 \%$ \\
\hline
\end{tabular}

\section{Acknowledgements}

This work was financially supported by the Foundation of Education Department of Heilongjiang Province (12541086).

\section{References}

[1] He Xin-gui,Xu Shao-hua.Process neural networks [M]. Science Press, 2007.6.

[2] Eusuff M M, Lansey K E. Optimization of Water DistributionNetwork Design Using the Shuffled Frog Leaping Algorithm[J].Journal of Water Sources Planning and Management, 2003, 129(3):210-225..

[3] S Rahnamayan, H R Tizhoosh, M A Salama. Opposition Versus randomness in softcomputing techniques. Applied Soft Computer.8(2):906-918,2008.

[4] FU Bin,LI Dao-guo,WANG Mu-kuai. Review and prospect on research of cloud model [J] . Application Research of Computers, 2011, 28(2):420- 426.

[5] ZHANG Guang-Wei, HE Rui,LIU Yu. An Evolutionary Algorithm Based on Cloud Model[J]. CHINESE JOURNAL OF COMPUTERS, 2008 , 31(7):1082-1090. 
[6] XU Xiao-bo, ZHENG Kang-feng, LI Dan. New chaos-particle swarm optimization algorithm[J]. Journal on Communications, 2012, 33(1):24-37. 